Bull. Austral. Math. Soc.

Vol. 40 (1989) [243-254]

\title{
PERMUTABLE WORD PRODUCTS IN GROUPS
}

\author{
P.S. Kim AND A.H. Rhemtulla \\ Dedicated to Professor B.H. Neumann \\ on his eightieth birthday
}

\begin{abstract}
Let $u\left(x_{1}, \ldots, x_{n}\right)=x_{11} \ldots x_{1 m}$ be a word in the alphabet $x_{1}, \ldots, x_{n}$ such that $x_{1 i} \neq$ $x_{1 i+1}$ for all $i=1, \ldots, m-1$. If $\left(H_{1}, \ldots, H_{n}\right)$ is an $n$-tuple of subgroups of a group $G$ then denote by $u\left(H_{1}, \ldots, H_{n}\right)$ the set $\left\{u\left(h_{1}, \ldots, h_{n}\right) \mid h_{i} \in H_{i}\right\}$. If $\sigma \in S_{n}$ then denote by $u_{\sigma}\left(H_{1}, \ldots, H_{n}\right)$ the set $u\left(H_{\sigma(1)}, \ldots, H_{\sigma(n)}\right)$. We study groups $G$ with the property that for each $n$-t uple $\left(H_{1}, \ldots, H_{n}\right)$ of subgroups of $G$, there is some $\sigma \in S_{n}, \sigma \neq 1$ such that $u\left(H_{1}, \ldots, H_{n}\right)=u_{\sigma}\left(H_{1}, \ldots, H_{n}\right)$. If $G$ is a finitely generated soluble group then $G$ has this property for some word $u$ if and only if $G$ is nilpotent-by-finite. In the paper we also look at some specific words $u$ and study the properties of the associated groups.
\end{abstract}

\section{INTRODUCTION}

Let $n$ be a fixed positive integer, $X=\left\{x_{1}, \ldots, x_{n}\right\}$ a set of $n$ symbols and $F=$ $F(X)$ the free group on $X$. Let $U=\left\{u_{1}, u_{2}, \ldots\right\}$ and $V=\left\{v_{1}, v_{2}, \ldots\right\}$ be non-empty sets of elements in $F$. Define the class $P(U, V)$ to consist of groups $G$ such that given an $n$-tuple $\left(g_{1}, \ldots, g_{n}\right)$ of elements in $G, u\left(g_{1}, \ldots, g_{n}\right)=v\left(g_{1}, \ldots, g_{n}\right)$ for some $u \in U$ and some $v \in V, v \neq u$. Some examples:

1.1. Let $U=\{u\}$ where $u\left(x_{1}, \ldots, x_{n}\right)=x_{1} x_{2} \ldots x_{n}$ and $V=\left\{u_{\sigma} \mid \sigma \in S_{n} \backslash 1\right\}$ where $S_{n}$ is the symmetric group of degree $n$ and

$$
u_{\sigma}\left(x_{1}, \ldots, x_{n}\right)=u\left(x_{\sigma(1)}, \ldots, x_{\sigma(n)}\right)=x_{\sigma(1)} x_{\sigma(2)} \ldots x_{\sigma(n)} .
$$

Then every group in $P(U, V)$ is finite-by-abelian-by-finite. Conversely every finite-byabelian-by-finite group is in $P(U, V)$ for some suitable $n$. This was shown by Curzio, Longobardi, Maj and Robinson in [2]. These groups are more commonly referred to as $P_{n}$-groups.

1.2. Let $u=u\left(x_{1}, \ldots, x_{n}\right)=x_{1} x_{2} \ldots x_{n}, U=\left\{u_{\sigma} \mid \sigma \in S_{n}\right\}$ and $V=U$. Then $P(U, V)$-groups, more commonly referred to as $Q_{n}$-groups or rewritable groups, are again finite-by-abelian-by-finite groups as shown by Blyth in [1]. That an abelian-by finite group is in $Q_{n}$ for some $n$ is implicit in Theorem 1 of Kaplansky [4].

Received 28 March 1989

Partial support by NSERC is gratefully acknowledged by the second author.

Copyright Clearance Centre, Inc. Serial-fee code: 0004-9729/89 \$A2.00+0.00. 
1.3. If we take $n=2, u=u\left(x_{1}, x_{2}\right)=\left(x_{1} x_{2}\right)^{r}, v=v\left(x_{1}, x_{2}\right)=\left(x_{2} x_{1}\right)^{r}$ where $r>0$ is fixed and $U=\{u\}, V=\{v\}$ then $G \in P(U, V)$ if and only if $G / Z(G)$ is of exponent $r$. This is not difficult to verify.

In general the classes $P(U, V)$ may be viewed as generalising varieties and, except for some specific sets $U$ and $V$, it is very difficult to describe them. We now turn to related classes of groups.

Let $n>0$ be fixed, $X=\left\{x_{1}, \ldots, x_{n}\right\}$ be a set of idempotent variables and $S=S(X)$ the free semigroup generated by $X$. Thus for any $u \in S, u=u\left(x_{1}, \ldots, x_{n}\right)=$ $x_{11} x_{12} \ldots x_{1 m}$ where $x_{1 i} \in X$ and $x_{1 i} \neq x_{1 i+1}$ for all $i=1, \ldots, m-1$. If $\left(H_{1}, \ldots H_{n}\right)$ is an $n$-tuple of subgroups of a group $G$ then denote by $u\left(H_{1}, \ldots, H_{n}\right)$ the set $\left\{u\left(h_{1}, \ldots, h_{n}\right) \mid h_{i} \in H_{i}\right\}$. Thus if $u\left(x_{1}, \ldots, x_{n}\right)$ is as above then

$$
u\left(H_{1}, \ldots, H_{n}\right)=H_{11} H_{12} \ldots H_{1 m}
$$

If $U$ and $V$ are sets of elements in $S$ then define the class $S P(U, V)$ to consist of groups $G$ such that for any $n$-tuple $\left(H_{1}, \ldots, H_{n}\right)$ of subgroups of $G, u\left(H_{1}, \ldots, H_{n}\right)=$ $v\left(H_{1}, \ldots, H_{n}\right)$ for some $u \in U$ and some $v \in V, v \neq u$. Some examples:

1.4. Let $U=\{u\}$ where $u\left(x_{1}, \ldots, x_{n}\right)=x_{1} x_{2} \ldots x_{n}$ and $V=\left\{u_{\sigma} \mid \sigma \in S_{n} \backslash 1\right\}$. As in $1.1, u_{\sigma}\left(x_{1}, \ldots, x_{n}\right)=u\left(x_{\sigma(1)}, \ldots, x_{\sigma(n)}\right)$. Finitely generated soluble $S P(U, V)$ groups are finite-by-abelian. Conversely every finite-by-abelian group is an $S P(U, V)$ group for some integer $n$. These results are contained in [6]. From [5] we know that periodic $S P(U, V)$-groups are locally finite. The structure of $S P(U, V)$-groups in general seems difficult to deternune.

1.5. For each positive inleger $r$ let $u_{r}=u_{r}(x, y)=(x y)^{r}$, and $v_{r}=v_{r}(x, y)=$ $(y x)^{r}$. Let $U=\left\{u_{r}, r=1,2, \ldots\right\}$ and $V=\left\{v_{r}, r=1,2, \ldots\right\}$. Then the class $S P(U, V)$ is precisely the class of groups in which every subgroup is elliptically embedded. Groups with this property are considered in $[\mathbf{7}, \mathbf{8}]$. It is known that a finitely generated soluble group $G$ is in this class if and only if it is finile-by-nilpotent. The same is true if we replace "soluble group" by "residually finite $p$-group" in the above statement.

In this paper we shall not look at $P(U, V)$-groups, but concentrate our attention on $S P(U, V)$-groups. The two main results are as follows:

Theorem 1. Let $G$ be a finitely generated soluble group, $U=\{u\}$ where $u=u\left(x_{1}, \ldots, x_{n}\right)=\left(x_{1} \ldots x_{n}\right)^{r}$ and $V=\left\{u_{\sigma} \mid \sigma \in S_{n}\right\}$ where $u_{\sigma}\left(x_{1}, \ldots, x_{n}\right)=$ $u\left(x_{\sigma(1)}, \ldots, x_{\sigma(n)}\right)$. Then $G$ is an $S P(U, V)$ group for some $n>1, r>0$ if and only if $G$ is finite-by-nilpotent.

ThEOREM 2. Let $U=\{u\}$ where $u$ is a word in idempotent variables $x_{1}, \ldots, x_{n}$, $n>1 ;$ and let $V=\left\{u_{\sigma} \mid \sigma \in S_{n}\right\}$. If $G$ is a finitely generated soluble group in $S P(U, V)$, then $G$ is nilpotent-by-finite. 
We can not replace "nilpotent-by-finite" in Theorem 2 by the stronger condition "finite-by-nilpotent" of Theorem 1. It is tedious, but we will show that the infinite dihedral group $D_{\infty}$ lies in $S P(U, V)$ where $U=\{u\}, V=\left\{u_{\sigma} \mid \sigma \in S_{n}\right\}$ and $u=u\left(x_{1}, x_{2}, x_{3}, x_{4}\right)=x_{1} x_{4} x_{2} x_{3} x_{2} x_{3} x_{4} x_{1}$ and it is well-known that $D_{\infty}$ is not finiteby-nilpotent.

At present little is known about the various classes $S P(U, V)$ and $P(U, V)$. The following questions stand a good chance of getting answered, at least partially, in the not too distant future!

QUESTION 1: Let $U$ and $V$ be finite sets of words in $S=S(X)$ where $X=$ $\left\{x_{1}, \ldots, x_{n}\right\}$ and let $G$ be a finitely generated soluble group in the class $S P(U, V)$. Is $G$ nilpotent-by-finite?

QUESTION 2: For which words $u$ in $S=S(X), X=\left\{x_{1}, \ldots, x_{n}\right\}$ are the periodic $S P(U, V)$ groups locally finile; where $U=\{u\}$ and $V=\left\{u_{\sigma}, \sigma \in S_{n}\right\}$.

QUESTION 3: Let $G$ be a finitely generated residually finite $p$-group, $p$ a prime. For which sets $U, V$ of words in $S=S(X), X=\left\{x_{1}, \ldots, x_{1}\right\}$; would $G \in S P(U, V)$ imply $G$ is nilpotent-by-finite?

Finally we ask if the classes $S P(U, V)$ can contain finitely generated infinite simple groups.

\section{Proofs}

Since the hypothesis of Theorem 1 is a more restricted form of the hypothesis of Theorem 2, it would be proper to prove Theorem 2 and then establish the extra property required in Theorem 1 . The reduction from soluble to nilpotent-by-finite will be achieved using two intermediate steps; these are dealt with in the following lemmas.

LEMMA 2.1. The wreath product of a cyclic group of order $p$ with the infinite cyclic group is not in the class $S P(U, V)$ where $U, V$ are as in the statement of Theorcm 2.

LeMma 2.2. Let $G=\langle A, t\rangle$ where $A$ is a torsion-free abelian group of finite rank on which $\langle t\rangle$ acts rationally irreducibly. If $G \in S P(U, V)$ where $U, V$ are as in the statement of Theorem 2 , then for some positive integer $k,\left\langle t^{k}\right\rangle$ acts trivially on $A$.

LEMMA 2.3. If $G=\langle A, t\rangle$, where $A \unlhd G$ and is abelian of finite rank, and $G \in$ $S P(U, V)$ where $U, V$ are as in the statement of Theorem 2 , then for some $\ell>0,\left\langle A, t^{\ell}\right\rangle$ has a non-trivial centre.

LEMMA 2.4. Let $G=\langle A, t\rangle$ where $A$ is a torsion-free abelian group of finite rank on which $\langle t\rangle$ acts rationally irreducibly. If $G \in S P(U, V)$ where $U, V$ are as in the statement of Theorem 1 , then $\langle t\rangle$ acts trivially on $A$. 
Proof of Theorem 2: By hypothesis, $X=\left\{x_{1}, \ldots, x_{n}\right\} u=u(X)=x_{11} x_{12} \ldots$ $x_{1 m}$ where $x_{1 i} \in X$ for all $i=1, \ldots, m$ and $x_{1 i} \neq x_{1 i+1}$ for all $i=1, \ldots, m-1$. Let $G$ be a finitely generated soluble group such that for any $n$-tuple $\left(H_{1}, \ldots, H_{n}\right)$ of subgroups of $G$, there is a permutation $\sigma \neq 1$ in $S_{n}$ such that

$$
u\left(H_{1}, \ldots, H_{n}\right)=H_{11} H_{12} \ldots H_{1 m}=u\left(H_{\sigma(1)}, \ldots, H_{\sigma(n)}\right)=H_{\sigma(11)} \ldots H_{\sigma(1 m)} .
$$

We need to show that $G$ is nilpotent-by-finite, and we proceed by induction on the solubility length of $G$. If $G$ is abelian then there is nothing to prove. Let $G$ be soluble of length $d$ and assume that the result holds for soluble groups of smaller length. Since the class $S P(U, V)$ is subgroup and quotient closed, we may suppose that $G$ has a normal abelian subgroup $A$ such that $G / A$ is nilpotent-by-finite. In particular $G$ is abelian by polycyclic. If $G$ does not have finite rank then it has a section isomorpluc to the wreath product of a cyclic gronp of prime order $p$ and the infinite cyclic group. This is not possible by Lemma 2.1. IIence we conclude that $G$ has finite rank.

As $G$ is finitely generated abelian-by-polycyclic, it satisfies the maximal condition for normal subgroups. If $G$ is not nilpotent-by-finite, then let $B$ be a maximal normal subgroup of $G$ such that $G / B$ is not nilpotent-by-finite. Now we replace $G$ by $G / B$ and hence assume that every proper quotient of $G$ is nilpotent-by-finite.

Let $T$ be the torsion subgroup of $A$. Then $T$ has finite rank and is of bounded exponent since $G$ satisfies the maximal condition for normal subgroups. Thus $T^{\prime}$ is finite, and $C=C_{G}\left(T^{\prime}\right)$, the centraliser of $T$ in $G$, is of finite index in $G$. If $T \neq 1$ then $G / T$ is nilpotent-by-finite and hence $C / T$ is nilpotent-by-finite. Since $T \leqslant Z(C)$, the centre of $C$, then $C$ and hence $G$ would be nilpotent-by-finite. Thus we assume $T=1$ and hence $A$ is torsion-free, and by passing to a suitable subgroup of finite index in $G$, if necessary, we may assume further that $G / A$ is a finitely generated torsion-free nilpotent group. 'Thus there exists a finite set $T=\left\{t_{1}, \ldots, t_{r}\right\}$ of elements in $G$ such that $G=\langle A, T\rangle$ and

$$
A=G_{0} \leqslant\left\langle G_{0}, t_{1}\right\rangle=G_{1} \leqslant \ldots \leqslant\left\langle G_{r-1}, t_{r}\right\rangle=G_{r}=G
$$

is a central series from $A$ to $G$ with torsion-free factors.

If $r=1$ then $G=\left\langle A, t_{1}\right\rangle$. By Lemma $2.3 Z\left(\left\langle A, t_{1}^{\ell_{1}}\right\rangle\right) \neq 1$ for some $\ell_{1}>0$ and hence $D=A \cap Z\left(\left\langle A, t_{1}^{\ell_{1}}\right\rangle\right)$ is a non-trivial normal subgroup of $G$. By our choice of $G, G / D$ is nilpotent-by-finite and hence $G$ is nilpotent-by-finite.

Now suppose we have established the result for the case $r<d$ and suppose $r=d$. Then $G_{d-1}$ is nilpolent-by-finite and $G=\left\langle G_{d-1}, t_{d}\right\rangle$. Let $H=\left\langle A, G_{d-1}^{\ell}\right\rangle$ for some suitable $\ell>0$ so that $H$ is nilpotent. Let $Y=A \cap Z(H)$ then $Y$ is normal in $\left\langle H, t_{d}\right\rangle$ which is of finite index in $G$. Moreover $Z\left(\left\langle Y, t_{d}^{\ell_{1}}\right\rangle\right) \neq 1$ for some $\ell_{1}>0$ by 
Lemma 2.3, so that $D_{1}=Y \cap Z\left(\left\langle Y, t_{d}^{\ell_{1}}\right\rangle\right)$ is a non-trivial subgroup of $G$ contained in the centre of $\left\langle H, t_{d}^{\ell_{1}}\right\rangle$ which is of finite index in $G$. We may replace $\left\langle H, t_{d}^{\ell_{1}}\right\rangle$ by its normal interior in $G$, if necessary; it still contains $A$ and hence $D_{1}$. Now $\left\langle H, t_{d}^{\ell_{1}}\right\rangle / D_{1}$ is uilpotent-by-finite, $D_{1} \leqslant Z\left(\left\langle H, t_{d}^{\ell_{1}}\right\rangle\right)$ and $\left\langle H, t_{d}^{\ell_{1}}\right\rangle$ is of finite index in $G$. Thus $G$ is nilpotent-by-finite, as required.

Proof of Theorem 1: Since the hypotheses of Theorem 2 are satisfied by the group of Theorem 1, we may assume $G$ to be finitely generated nilpotent-by-finite. Let $T$ be the maximal finite normal subgroup of $G$. Since we wish to show that $G$ is finite-by-nilpotent we may look at $G / T$, if necessary, and hence assume that $G$ has no non-trivial finite normal subgroup. Let $F$ be the Fitting subgroup of $G$. If $F \neq G$ then pick any $t \in G \backslash F$ such that $t^{p} \in F$. Clearly it is sufficient to show that $\langle F, t\rangle$ is nilpotent for $G / F$ is finite and soluble, we can reach $G$ from $F$ by a subnormal series with factors of prime order. Thus we assume $G=\langle F, t\rangle, t^{p} \in F$ and $F$ is torsion-free.

Let $H$ be the hypercentre of $G$. Then $H \cap F$ is isolated in $F$. This may be seen by first checking it for $Z(G) \cap F$ and then by taking the quotient of $G$ by this sulbgroup, and using induction. Observe that if $H \nless F$ then $G=H F$ and $G$ is nilpotent. So assume $I I \leqslant F$. Next we look at $G / H$. If $G / H$ is nilpotent then so is $G$. So we assume $H=1$. Let $A$ be a non-trivial normal subgroup of $G$ of least Hirsch length and $A \leqslant Z(F)$. Since $\langle A, t\rangle \in S P(U, V),\langle t\rangle$ acts trivially on $A$ by Lemma 2.4. Thus $A \leqslant Z(G)$ contradicting the assumption that $Z(G)=1$. This concludes the proof that if $G \in S P(U, V)$ then $G$ is finite-by-nilpotent.

Now suppose that $G$ is a finitely generated finite-by-nilpotent group. For any subgroup $L$ of $G$ let $\gamma(L)$ denote the nilpotent residual of $L$. Thus $\gamma(L)$ is the intersection of the terms of the lower central series of $L$. Let $F=\gamma(G)$. It is finite by hypothesis and $G / F$ is nilpotent of class $c_{1}$ for some $c_{1}>0$. Thus $\gamma(L)=\gamma_{c}(L)$ for all $L \leqslant G$ where $c=|F|+c_{1}$. We show, by induction on $|\gamma\langle H, K\rangle|=s$, that $(H K)^{d_{s}}=(K H)^{d_{s}}=\langle H, K)$ for all subgroups $H, K$ of $G$ where $d_{1}=(4 r)^{c}, r=$ rank of $G ; d_{i}=d_{i-1}+2 i\left(i+d_{1}\right), i>1$. In particular $(H K)^{d}=(K H)^{d}=\langle H, K\rangle$ for all $H, K$ where $d=d_{f}$, and $f=|F|$.

By Proposition 2 of $[7], \Gamma(H K)^{t}=\Gamma(K I I)^{t}=\langle K, H\rangle$ where $\Gamma=\gamma\langle K, H\rangle, t=$ $(4 r)^{c}$, and $r$ is the rank of $G$. Thus if $\Gamma=1$ then $d_{1}=t$ will suffice.

For any $a \in(H K)^{t}, a=g b$ for some $g \in \Gamma$ and $b \in(K H)^{t}$ so that $a b^{-1}=$ $g \in \Gamma \cap(H K)^{2 t}$. II $\Gamma \cap(K H)^{2 t}=1$, then $a=b$ and $(H K)^{t}=(K H)^{t}$. This implies $\langle H, K\rangle=(H K)^{t}$, and again $d_{1}=t$ suffices.

If $\Gamma_{1}=\Gamma \cap(H K)^{2 t} \neq 1$, then for each integer $m \geqslant 1$ let $\Gamma_{m+1}=\Gamma_{m} \cup \Gamma_{m}^{H K}$ so that $\Gamma_{m} \subseteq(H K)^{2 t+2 m}$. Observe that $\Gamma_{m}=\Gamma_{m+1}$ implies $\left\langle\Gamma_{m}\right\rangle=\left\langle\Gamma_{m}^{H}\right\rangle=\left\langle\Gamma_{m}^{K}\right\rangle$. Since $\Gamma_{m} \subseteq \Gamma$ and $\left|I^{\prime}\right|=s, \Gamma_{s}=\Gamma_{s+1}$. Also note that $\left\langle\Gamma_{m}\right\rangle \subseteq \Gamma_{m}^{s}$. Thus the normal closure 
$N$ of $\Gamma_{1}$ in $\langle H, K\rangle$ lies in $(H K)^{\lambda}$ where $\lambda=\lambda_{0}=2\left(s^{2}+t s\right)$.

Now $N H$ and $N K$ both lie in $(H K)^{\lambda}$ and $(N H N K)^{m} \subseteq(H K)^{\lambda+m}$ for all $m>0$. Rank of $\langle H, K\rangle / N$ is no greater than $r, \gamma(\langle H, K\rangle / N)=\gamma_{c}(\langle H, K\rangle / N)$ and $|\gamma(\langle H, K\rangle / N)|<|\gamma(\langle H, K\rangle)|$. Thus by the induction hypothesis, $N(H K)^{d^{\prime}}=$ $N(K H)^{d^{\prime}}=\langle H, K\rangle$ where $d^{\prime}=t+\lambda_{2}+\cdots+\lambda_{s-1}=d_{s-1}$. Since $(H K)^{\lambda} \geqslant N$, we obtain $(H K)^{d_{s}}=(K H)^{d_{s}}=\langle H, K\rangle$ where $d_{s}=d_{s-1}+\lambda_{s}$.

Now that we have shown that for a finitely generated finite-by-nilpotent group $G$ there is an integer $d$ such that $(H K)^{d}=(K H)^{d}$ for all subgroups $H, K$ of $G$, we let $u=u(x, y)=(x y)^{d}, v=v(x, y)=(y x)^{d}$ then $G \in S P(u, v)$. This completes the proof of the second part of the theorem.

Proof of Lemma 2.1: Let $G$ be the wreath product of a cyclic group of order $p$ and an infinite cyclic group $\langle t\rangle$. Then we can identify each element of $G$ by a pair $\left(f(t), t^{\alpha}\right)$ where $f(t) \in F_{p}\langle t\rangle$, the additive group of the group ring of the infinite cyclic group $\langle t\rangle$ over the field $F_{p}$ of $p$ elements, and $\alpha \in Z$. The product of two such elements is then given by the rule: $\left(f(t), t^{\alpha}\right)\left(g(t), t^{\beta}\right)=\left(f(t)+t^{\alpha} \cdot g(t), t^{\alpha+\beta}\right)$. The elements of the base group correspond to those pairs where $\alpha=0$ and the elements of the top group correspond to those pairs where $f(t)=0$.

We are given $X=\left\{x_{1}, \ldots, x_{n}\right\}, u=u(X)=x_{11} x_{12} \ldots x_{1 m} ; x_{1 i} \in X, \quad x_{1 i} \neq$ $x_{1 i+1}, i=1, \ldots, m-1$ and we are required to show that there exist subgroups $H_{1}, \ldots, H_{n}$ of $G$ such that $H_{11} H_{12} \ldots H_{1 m} \neq H_{\phi(11)} H_{\phi(12)} \ldots H_{\phi(1 m)}$ for any $\phi \neq 1$ in $S_{n}$.

Take $H_{i}=\left\langle h_{i}\right\rangle$ where $h_{i}=\left(\left(1-t^{\alpha_{i}}\right) f_{i}, t^{\alpha_{i}}\right) ; f_{i}=f_{i}(t)$ and $\alpha_{i}$ are to be chosen appropriately. Note that $h_{i}^{k}=\left(\left(1-t^{k \alpha_{i}}\right) f_{i}, t^{k \alpha_{i}}\right)$, and a general element of $u\left(H_{1}, \ldots, H_{n}\right)$ is $h_{11}^{k_{1}} \ldots h_{1 m}^{k_{m}}=$

$$
\begin{aligned}
& \left(\left(1-t^{k_{1} \alpha_{11}}\right) f_{11}, t^{k_{1} \alpha_{11}}\right) \ldots\left(\left(1-t^{k_{m} \alpha_{1 m}}\right) f_{1 m}, t^{k_{m} \alpha_{1 m}}\right) \\
& \left(\left(1-t^{k_{1} \alpha_{11}}\right) f_{11}+t^{\lambda_{1}}\left(1-t^{k_{2} \alpha_{12}}\right) f_{12}+\cdots+t^{\lambda_{m-1}}\left(1-t^{k_{m} \alpha_{1 m}}\right) f_{1 m}, t^{\lambda_{m}}\right)
\end{aligned}
$$

where $\lambda_{i}=k_{1} \alpha_{11}+\cdots+k_{i} \alpha_{1 i}, \quad i=1, \ldots, m$. Partition the set $\{1, \ldots, m\}$ as the union $S_{1} \cup \ldots \cup S_{n}$ where $S_{i}=\left\{j \mid x_{i j}=x_{i}\right\}$. Then a general element of $u\left(H_{1}, \ldots, H_{n}\right)$ is of the form $\left(\sum_{i=1}^{n}\left(f_{i} \sum_{j \in S_{i}}\left(t^{\lambda_{j-1}}-t^{\lambda_{j}}\right)\right), t^{\lambda_{m}}\right)$ with the understanding that $\lambda_{0}=0$. Likewise $u\left(H_{\sigma(1)}, \ldots, H_{\sigma(n)}\right)$ consists of elements of the form $\left(\sum_{i=1}^{n}\left(f_{\phi(i)} \sum_{j \in S_{i}}\left(t^{\mu_{j-1}}-t^{\mu_{j}}\right)\right), t^{\mu_{m}}\right)$ where $\mu_{i}=\ell_{1} \alpha_{\phi(11)}+\cdots+\ell_{i} \alpha_{\phi(1 i)}, i=1, \ldots, m$ and $\mu_{0}=0$. If $\sigma$ denotes the inverse 
of $\phi$, then we may write these elements as $\left(\sum_{i=1}^{n}\left(f_{i} \sum_{j \in S_{\sigma(i)}}\left(t^{\mu_{j-1}}-t^{\mu_{j}}\right)\right), t^{\mu_{m}}\right)$.

Now $\quad\left(\sum_{i=1}^{n}\left(f_{i} \sum_{j \in S_{i}}\left(t^{\lambda_{j-1}}-t^{\lambda_{j}}\right)\right), t^{\lambda_{m}}\right)=\left(\sum_{i=1}^{n}\left(f_{i} \sum_{j \in S_{\sigma(i)}}\left(t^{\mu_{j-1}}-t^{\mu_{j}}\right)\right), t^{\mu_{m}}\right)$

implies $\lambda_{m}=\mu_{m}$ and

$$
\sum_{i=1}^{n} f_{i}\left(\sum_{j \in S_{i}}\left(t^{\lambda_{j-1}}-t^{\lambda_{j}}\right)\right)=\sum_{i=1}^{n} f_{i}\left(\sum_{j \in S_{\sigma(i)}}\left(t^{\mu_{j-1}}-t^{\mu_{j}}\right)\right)
$$

Let $p_{1}, \ldots, p_{n}$ be distinct primes, each greater than $m$. Put $p=p_{1} \ldots p_{n}, k_{i}=1$ and $\alpha_{i}=p / p_{i}, i=1, \ldots, n$. Then for each $i>0, \lambda_{i}=\alpha_{11}+\cdots+\alpha_{1 i}$. Let $f_{i}=t^{p^{i}}$. Note that $\lambda_{j}<p$ for all $j$ and they are all distinct. Also note that $f_{i} t^{\lambda_{j}}=f_{i^{\prime}} t^{\mu^{\prime}}$ implies $p^{i}+\lambda_{j}=p^{i^{\prime}}+\mu_{j^{\prime}}$. Ilence $\mu_{j^{\prime}} \equiv \lambda_{j} \bmod p$ so that $\mu_{j^{\prime}} \neq \lambda_{i} \bmod p$ for any $i \neq j$. Thus each $\lambda_{j}$ is congruent modulo $p$ to precisely one $\mu_{j^{\prime}}$.

Now $1 \in S_{\sigma(k)}$ for some $k$. Thus $f_{k}\left(t^{\mu_{0}}-t^{\mu_{1}}\right)$ is a term on the right hand side of (1). Since $\mu_{0}=0$ and the only $\lambda_{i}$ equal to zero is $\lambda_{0}, f_{k}\left(t^{\lambda_{0}}-t^{\lambda_{1}}\right)$ appears on the left hand side of (1). In particular $1 \in S_{k}$. Since $S_{1}, \ldots, S_{n}$ partition the set $\{1, \ldots, m\}$ and $1 \in S_{k} \cap S_{\sigma(k)}$, it follows that $\sigma(k)=k$. Hence $\mu_{1}$ and $\lambda_{1}$ are both congruent to zero $\bmod p / p_{k}$; it follows that $\mu_{1}=\lambda_{1}$.

Suppose, by way of induction, that we have established that $\mu_{j}=\lambda_{j}$ for all $j<e$. Then $\mu_{e}-\mu_{e-1}=\ell_{e} \alpha_{\phi(1 e)}$ which is congruent to zero mod all primes $p_{i}$ except possibly one namely. $p_{\phi(1 e)}$. Now we look at $\left\{\lambda_{j}-\lambda_{e-1}, j=e, \ldots, m\right\} . \lambda_{e}-\lambda_{e-1}=\alpha_{1 e}$ is congruent to zero $\bmod$ all primes $p_{i}, p_{i} \neq p_{1 e}$. For each of the other $\lambda_{j}-\lambda_{e-1}$, we can find at least two primes amongst $\left\{p_{1}, \ldots, p_{n}\right\}$ such that $\lambda_{j}-\lambda_{e-1}$ is not congruent to zero mod either of them. Hence $0 \not \equiv \alpha_{1 e} \equiv \ell_{e} \alpha_{\phi(1 e)} \bmod p_{1 e}$. But $\alpha_{\phi(1 e)} \neq \alpha_{1 e}$ implies $\alpha_{\phi(1 e)} \equiv 0 \bmod p_{1 e}$. Thus $\alpha_{\phi(1 e)}=\alpha_{1 e}$, and $x_{1 e}=x_{\phi(1 e)}=x_{k^{\prime}}$, say. Thus $\sigma\left(k^{\prime}\right)=k^{\prime}$ and $e \in S_{k^{\prime}}$. Thus $f_{k^{\prime}}\left(t^{\mu_{e-1}}-t^{\mu_{e}}\right)=f_{k^{\prime}}\left(t^{\lambda_{e-1}}-t^{\lambda_{e}}\right)$ and $\lambda_{e}=\mu_{e}$.

It is now clear that $\phi(j)=j$ for all $j=1, \ldots, n$ and hence $\phi$ is the identity permutation of the set $\{1, \ldots, n\}$ as required.

Proof of Lemma 2.2: We are given a group $G=\langle A, t\rangle$ where $A$ is torsionfree abelian of finite rank on which $\langle t\rangle$ acts rationally irreducibly. Let us assume, if possible, that $[A, t] \neq 1$. Then $V=A \otimes_{\mathrm{Z}} \mathrm{Q}$ is an irreducible $\mathrm{Q}\langle t\rangle$-module and by Schur's Lemma, the centraliser ring $\Gamma=$ End $_{Q\langle t\rangle} V$ is a division ring of finite dimension over $Q$. The innage of $\langle t\rangle$ in End $Q$ clearly lies in and spans $\Gamma$ so that $\Gamma$ is an algebraic number field. Moreover, regarded as a $\Gamma$-space, $V$ is one-dimensional. Thus we may consider $A$ to be an additive subgroup of $Q(\tau)$ for some algebraic number $\tau$ and the action of conjugation by $t$ as multiplication by $\tau$. 
Let $h_{i}=b_{i}\left(1-\tau^{\alpha_{i}}\right) t^{\alpha_{i}}$ for suitable integer $\alpha_{i}$ and $b_{i}\left(1-\tau^{\alpha_{i}}\right) \in A$. Let $H_{i}=\left\langle h_{i}\right\rangle$. Note that $h_{i}^{k}=b_{i}\left(1-\tau^{k \alpha_{i}}\right) t^{k \alpha_{i}}$.

As in Lemma 2.1, we are given $u=u\left(x_{1}, \ldots, x_{n}\right)=x_{11} x_{12} \ldots x_{1 m} ; x_{1 i} \epsilon$ $\left\{x_{1}, \ldots, x_{n}\right\}, x_{1 i} \neq x_{1 i+1}, \quad i=1, \ldots, m-1$; and we need to show that with proper chuice of $b_{i}$ and $\alpha_{i}$, subgroups $H_{1}, \ldots, H_{n}$ can be found such that $H_{11} \ldots H_{1 m} \neq$ $H_{\phi(11)} \ldots H_{\phi(1 m)}$ for any $\phi \neq 1$ in $S_{n}$. Now

$$
\begin{aligned}
h_{11}^{k_{1}} \ldots h_{1 m}^{k_{m}} & =b_{11}\left(1-\tau^{k_{1} \alpha_{11}}\right) t^{k_{1} \alpha_{11}} \ldots b_{1 m}\left(1-\tau^{k_{m} \alpha_{1 m}}\right) t^{k_{m} \alpha_{1 m}} \\
& =b_{11}\left(1-\tau^{\lambda_{1}}\right)+b_{12}\left(\tau^{\lambda_{1}}-\tau^{\lambda_{2}}\right)+\cdots+b_{1 m}\left(\tau^{\lambda_{m-1}}-\tau^{\lambda_{m}}\right) t^{\lambda_{m}} \\
\lambda_{i} & =k_{1} \alpha_{11}+\cdots+k_{i} \alpha_{1 i}, \quad i=1, \ldots, m .
\end{aligned}
$$

where

We shall put $\lambda_{0}=0$ and write $1=\tau^{0}=\tau^{\lambda_{0}}$.

Thus a general element of $u\left(H_{1}, \ldots, H_{n}\right)$ has the form

$$
\sum_{i=1}^{n}\left(b_{i} \sum_{j \in S_{i}}\left(\tau^{\lambda_{j-1}}-\tau^{\lambda_{j}}\right)\right) t^{\lambda_{m}}
$$

where $S_{i}=\left\{j ; x_{1 j}=x_{i}\right\}$ so that $\{1, \ldots, m\}$ is the disjoint union of $S_{1}, \ldots, S_{n}$. Likewise the general element of $u\left(H_{\phi(1)} \ldots H_{\phi(n)}\right)$ has the form

$$
\sum_{i=1}^{n}\left(b_{i} \sum_{j \in S_{\sigma(i)}}\left(\tau^{\mu_{j-1}}-\tau^{\mu_{j}}\right)\right) t^{\mu_{m}}
$$

where $\mu_{i}=\ell_{1} \alpha_{\phi(1)}+\cdots+\ell_{i} \alpha_{\phi(1 i)}, \quad i=1, \ldots, m ; \mu_{0}=0$ and $\sigma=\phi^{-1}$. This is shown in the same way as in the proof of Lemma 2.1. In particular $\mu_{m}=\lambda_{m}$ and

$$
\sum_{i=1}^{n} b_{i}\left(\sum_{j \in S_{i}}\left(\tau^{\lambda_{j-1}}-\tau^{\lambda_{j}}\right)-\sum_{j \in S_{\sigma(i)}}\left(\tau^{\mu_{j-1}}-\tau^{\mu_{j}}\right)\right)=0
$$

Now we return to pick $b_{i}$ and $\alpha_{i}$ appropriately. For each integer $r>1$, pick primes $p_{r 1}, \ldots, p_{r n}$ to salisfy $2^{r}<p_{r 1}$ and $p_{r i}^{2}<p_{r i+1}, \quad i=1, \ldots, n-1$. Put $q_{r}=$ $p_{r 1} \ldots p_{r n}, \quad b_{r i}(y)=y^{q_{r}^{i}}$ and $\alpha_{r i}=q_{r} / p_{r i}, \quad i=1, \ldots, n$. To make the notation simpler, we shall write $b_{i}$ for $b_{r i}$ and $\alpha_{i}$ for $\alpha_{r i}$, where there is no ambiguity. Since there are infinitely many choices of $q_{r}$ and each choice of $q_{r}$ determines the sequence $H_{1}, \ldots, H_{n}$ of subgroups which in turn corresponds to some permutation $\phi \neq 1$ such that $u\left(H_{1}, \ldots, H_{n}\right)=u\left(H_{\phi(1)}, \ldots, H_{\phi(n)}\right)$, there is an infinite number of choices of $r$ such that $q_{r}$ correspond to the same pcrmutation $\phi$. 
If, for some value of $r$, we have the following stronger version of (2):

where

$$
\sum_{i=1}^{n} b_{i}(y) L_{i}(y)-\sum_{i=1}^{n} b_{i}(y) M_{i}(y)=0
$$

$$
L_{i}(y)=\sum_{j \in S_{i}}\left(y^{\lambda_{j-1}}-y^{\lambda_{j}}\right), \quad M_{i}(y)=\sum_{j \in S_{\sigma(i)}}\left(y^{\mu_{j-1}}-y^{\mu_{j}}\right)
$$

and $y$ is an indeterminant; then $\mu_{j}=\lambda_{j}$ for all $j$ and $\phi=1$. This is seen using arguments similar to those in the proof of Lemma 2.1. Thus we may suppose that for every $r$,

$$
P(y)=\sum_{i=1}^{n} b_{i}(y) L_{i}(y)-\sum_{i=1}^{n} b_{i}(y) M_{i}(y)
$$

is not zero but $P(\tau)=0$. If $Q(y)$ is any non-trivial segment of $P(y)$ such that $Q(\tau)=0$, then $Q^{\prime}(y)=P(y)-Q(y)$ is a segment of $P(y)$ with $Q^{\prime}(\tau)=0$. Moreover one or both of $Q(y)$ or $Q^{\prime}(y)$ contains at least as many monomials from $\sum_{i=1}^{n} b_{i}(y) L_{i}(y)$ as from $\sum_{i=1}^{n} b_{i}(y) M_{i}(y)$. Let $Q(y)$ be such a segment of $P(y)$ of shortest length. Thus

I. $Q(y) \neq 0$

II. $Q(\tau)=0$

III. $Q(y)$ contains al least as many monomials from $\sum_{i=1}^{n} b_{i}(y) L_{i}(y)$ as from $\sum_{i=1}^{n} b_{i}(y) M_{i}(y)$ and

IV. No proper segment has properties I and II.

We write $Q(y)=Q_{1}(y)-Q_{2}(y)$ where $Q_{1}(y)$ is a segment $\sum_{i=1}^{u} \pm y^{\lambda_{i}^{\prime}}$ of $\sum_{i=1}^{n} b_{i}(y) L_{i}(y)$, $Q_{2}(y)$ a segment $\sum_{i=1}^{v} \pm y^{\mu_{i}^{\prime}}$ of $\sum_{i=1}^{n} b_{i}(y) M_{i}(y)$ and we may suppose that there is no term in $Q_{1}(y)$ equal to any term in $Q_{2}(y)$. If $Q_{1}(y)$ has only one term in it, then $Q(y)= \pm y^{\lambda}$ or $\pm y^{\lambda} \pm y^{\mu}$ where $0 \neq \lambda$ and $\mu \neq \lambda$. In both cases $Q(\tau)=0$ implies $\tau$ is a root of unity and $\left[t^{k}, A\right]=1$ for some $k>0$, as required.

We may therefore assume that $Q_{1}(y)=\sum_{i=1}^{u} \pm y^{\lambda_{i}^{\prime}}$ has more than one term; $\lambda_{i}^{\prime}=$ $q^{i^{\prime}}+\lambda_{j_{i}}$ and $0 \leqslant \lambda_{1}^{\prime}<\cdots<\lambda_{u}^{\prime}$. Sinuilarly $Q_{2}(y)=\sum_{i=1}^{\nu} \pm y^{\mu_{i}^{\prime}}$ where $\mu_{1}^{\prime} \leqslant \ldots \leqslant \mu_{v}^{\prime}$. Let

$$
\nu_{1}=\min \left\{\lambda_{1}^{\prime}, \mu_{1}^{\prime}\right\} \quad \text { and } \quad \nu_{2}=\max \left\{\lambda_{u}^{\prime}, \mu_{v}^{\prime}\right\}
$$

Then $y^{-\nu_{1}} \cdot Q(y)$ is a polynomial of degree $\nu_{2}-\nu_{1}$ with non-zero constant term. Moreover $\nu_{2}-\nu_{1} \geqslant \lambda_{u}^{\prime}-\lambda_{1}^{\prime}>2^{r}$. Thus the degree of the polynomial increases with $r$, 
and hence there are infinitely many expressions

$$
1=\sum_{i=1}^{u+v} \varepsilon_{i} \tau^{\gamma_{i}}
$$

where $\gamma_{i} \geqslant 0, \varepsilon_{i} \in\{-1,0,1\}$ and no subsum of the right hand side of the equation is zero. But this is not possible by 'Theorem 1 of [9] which we state below for convenience. This completes the proof.

Tineorem. (Van Der Poorten) Let $K$ be a field of characteristic zero and II a finitely generated subgroup of the multiplicative group of $K$. Then for each integer $m>0$ there are only finitely many relations $u_{1}+\cdots+u_{m}=1$ with each $u_{i} \in H$ and no subsum of the left hand side is zero.

A result very similar to the above was proved by Evertse in [3]. One can avoid using the above deep result and use Lemma 1 and 2 of [7] and modify the argument slightly to get the required contradiction.

Proof of LEMMA 2.3: If the torsion subgroup of $A$ is non-trivial then it has a non-trivial normal subgroup $A_{1}$ of exponent $p$ for some prime $p$. This is finite since $A$ has finite rank, hence it is centralised by $t^{\ell}$ for some $\ell>0$ and $A_{1}$ lies in the centre of $\left\langle A, t^{\ell}\right\rangle$. We may thus assume that $A$ is torsion-free. Let $D$ be a non-trivial subgroup of $A$ of least rank subject to $D \unlhd G$. Lemma 2.2 applies to $\langle D, t\rangle$ and we conclude that $\left\langle D, t^{k}\right\rangle$ is abelian for some $k>0$. Ilence $D$ lies in the centre of $\left\langle A, t^{k}\right\rangle$.

Proof of LEMma 2.4: As the hypothesis of Theorem 1 is stronger than that of Theorem 2, Lemma 2.2 and its proof applies. We follow the proof of Lemma 2.2 and reach the situation where we may assuine $A$ to be an additive subgroup of $Q(\tau)$ for some algebraic number $\tau$ and the action of $t$ under conjugation is that of multiplication by $\tau$. Furthermore we may assume $\tau$ to be a primitive $k$ th root of unity and we need to show that $\tau=1$.

Let $h_{i}=\left(k^{i}(1-\tau), t^{-1}\right)$, and $I_{i}=\left\langle h_{i}\right\rangle$. Observe that $h_{i}^{\lambda}=\left(k^{i}\left(1-\tau^{\lambda}\right), t^{-\lambda}\right)$ and $h_{i}^{k}=t^{-k}$. Let $X=\left(I_{1} \ldots H_{n}\right)^{r}$ and suppose that for some $\phi \neq 1$ in $S_{n}, X=$ $\left(H_{\phi(1)} \ldots H_{\phi(n)}\right)^{r}$. If $\phi(1) \neq 1$, then $\left\langle H_{1}, H_{\phi(1)}\right\rangle \subseteq X$. But this is not possible since $X$ is the union of a finite number of cosets of $\left\langle t^{k}\right\rangle$ whereas $\left\langle H_{1}, H_{\phi(1)}\right\rangle$ contains the sulogroup generated by $\left(k^{\phi(1)}-k\right)(1-\tau)$, an infinite cyclic subgroup of $Q(\tau)$, not conlained in any finite union of cosets of $\left\langle t^{k}\right\rangle$. Hence $\phi(1)=1$ and similarly $\phi(n)=n$.

For any permutation $\pi$ in $S_{n}$, a typical element $x$ of $H_{\pi(1)} \ldots H_{\pi(n)}$ has the form

$$
\begin{aligned}
x & =h_{\pi(1)}^{\lambda_{1}} \ldots h_{\pi(n)}^{\lambda_{n}} \\
& =\left(k^{\pi(1)}\left(1-\tau^{\lambda_{1}}\right)+k^{\pi(2)}\left(1-\tau^{\lambda_{2}}\right) \tau^{\lambda_{1}}+\cdots+k^{\pi(n)}\left(1-\tau^{\lambda_{n}}\right) \tau^{\lambda_{1}+\cdots+\lambda_{n-1}}, t^{-\mu}\right)
\end{aligned}
$$


where $\mu=\lambda_{1}+\cdots+\lambda_{n}$ and $\lambda_{i}$ are arbitrary integers. In turn these elements may be written as

$$
\left(k^{\pi(1)}\left(1-\tau^{\alpha_{1}}\right)+k^{\pi(2)}\left(\tau^{\alpha_{1}}-\tau^{\alpha_{2}}\right)+\cdots+\ldots k^{\pi(n)}\left(\tau^{\alpha_{n-1}}-\tau^{\alpha_{n}}\right), t^{-\alpha_{n}}\right)
$$

where $\alpha_{i}$ are arbitrary integers. In particular $t^{-\alpha_{0}} x$ has the form $\left(h, t^{-\alpha_{n}}\right)$ where

$$
\begin{aligned}
h & =k^{\pi(1)}\left(\tau^{\alpha_{0}}-\tau^{\alpha_{1}}\right)+k^{\pi(2)}\left(\tau^{\alpha_{1}}-\tau^{\alpha_{2}}\right)+\cdots+k^{\pi(n)}\left(\tau^{\alpha_{n-1}}-\tau^{\alpha_{n}}\right) \\
& =\left(k^{\pi(1)} \tau^{\alpha_{0}}+\tau^{\alpha_{1}}\left(k^{\pi(2)}-k^{\pi(1)}\right)+\cdots+\tau^{\alpha_{n-1}}\left(k^{\pi(n)}-k^{\pi(n-1)}\right)-\tau^{\alpha_{n}} k^{\pi(n)} .\right.
\end{aligned}
$$

Thus, if $\alpha_{0}$ is a given fixed integer, the real part of $h$ is maximised by choosing $\alpha_{i} \equiv 0(\bmod k)$ if $\pi(i+1)>\pi(i), \alpha_{i} \equiv q(\bmod k)$ where $q=[k / 2]$ if $\pi(i+1)<\pi(i)$; $i=1, \ldots n-1$ and $\alpha_{n} \equiv q(\bmod k)$. If $\pi$ is the identity permutation then this value is $k \cos \left(2 \pi \alpha_{0}\right) /(k)+\left(k^{n}-k\right)-k^{n} \cos (2 \pi q) / k$.

On the other hand if $\pi \neq 1$ and $\pi(1)=1, \pi(n)=n$, then the maximum real part of the value of $h$ is

$$
k \cos \frac{2 \pi \alpha_{0}}{k}+\left(k^{n}-k\right)-k^{n} \cos \frac{2 \pi q}{k}+\sum\left(k^{\pi(i)}-k^{\pi(i+1)}\right)\left(1-\cos \frac{2 \pi q}{k}\right)
$$

where the sum is over all values of $i$ such that $\pi(i)>\pi(i+1)$. This value is clearly greater than the value obtained for the identity permutation $\pi$.

Now the general element of $\left(H_{\pi(1)} \ldots H_{\pi(n)}\right)^{r}$ is $\left(h, t^{-\alpha}\right)$ where $h$ is expressible in the form

$$
\sum_{i=1}^{r} k^{\pi(1)} \tau^{\alpha_{i 0}}+\tau^{\alpha_{i 1}}\left(k^{\pi(2)}-k^{\pi(1)}\right)+\cdots+\tau^{\alpha_{i n-1}}\left(k^{\pi(n)}-k^{\pi(n-1)}\right)-\tau^{\alpha_{i n}} k^{\pi(n)}
$$

where $\alpha_{10}=0, \alpha_{i n}=\alpha_{i+10}, i=1, \ldots, r-1, \quad \alpha_{r n}=\alpha$. By picking the values for $\alpha_{i j}$ to maximise the real part of $h$ as above, it is clear that the value achieved when $\pi \neq 1$ is greater than for $\pi=1$. Thus $\left(H_{\phi(1)} \ldots H_{\phi(n)}\right)^{r}, \phi(1)=1, \phi(n)=n, \phi \neq 1$, contains elements not contained in $\left(H_{1} \ldots H_{n}\right)^{r}$. This completes the proof.

\section{EXAMPLE}

Let $U=\{u\}$ where $u=u\left(x_{1}, \ldots, x_{4}\right)=x_{1} x_{4} x_{2} x_{3} x_{2} x_{3} x_{4} x_{1}$ and $V=\left\{u_{\sigma} \mid\right.$ $\left.\sigma \in S_{4}\right\}$. Then the infinite dihedral group $G$ is in $S P(U, V)$.

Consider $u\left(H_{1}, H_{2}, I_{3}, H_{4}\right)$ for given sulgroups $H_{1}, H_{2}, H_{3}, H_{4}$ of $G$. If $H_{1}$ or $H_{4}$ is normal in $G$ then $u\left(H_{1}, H_{2}, H_{3}, H_{4}\right)=u\left(H_{4}, H_{2}, H_{3}, H_{1}\right)$. If $H_{2}$ or $H_{3}$ is normal in $G$ then $u\left(H_{1}, H_{2}, H_{3}, H_{4}\right)=u\left(H_{1}, H_{3}, H_{2}, H_{4}\right)$. So assume none of the $H_{i}$ 's is normal in $G$. If for some $i, H_{i}$ is not of order two, then it contains a subgroup $K_{i}$ normal 
in $G$ and of index two in $H_{i}$. Moreover $u\left(H_{\sigma(1)} \ldots H_{\sigma(4)}\right)=K_{i} u\left(H_{\sigma(1)} \ldots H_{\sigma(4)}\right)$ for all $\sigma \in S_{n}$, and we may replace $G$ by $G / K_{i}$ and each of $H_{j}$ by $H_{j} K_{i} / K_{i}$. Thus the essential case to be considered is one where each $H_{i}$ is of order two.

Now $G=\langle a, t\rangle$ where $a^{t}=a^{-1}$ and $t^{2}=1 . H_{i}=\left\langle a^{\lambda_{i}} t\right\rangle, i=1,2,3,4$. We will show that the set $L=\mathrm{H}_{4} \mathrm{H}_{2} \mathrm{H}_{3} \mathrm{H}_{2} \mathrm{H}_{3} \mathrm{H}_{4}$ equals the set $\mathrm{R}=\mathrm{H}_{4} \mathrm{H}_{3} \mathrm{H}_{2} \mathrm{H}_{3} \mathrm{H}_{2} \mathrm{H}_{4}$. From this it follows that $u\left(H_{1}, H_{2}, H_{3}, H_{4}\right)=H_{1} L I H_{1}=H_{1} R H_{1}=u\left(H_{1}, H_{3}, H_{2}, H_{4}\right)$.

Now $a^{\lambda} \in H_{2} H_{3} H_{2} H_{3}$ if and only if $\lambda=0, \lambda_{2}-\lambda_{3}, \lambda_{3}-\lambda_{2}$ or $2 \lambda_{2}-2 \lambda_{3}$. $a^{\lambda} t \in H_{2} H_{3} H_{2} H_{3}$ if and only if $\lambda=\lambda_{2}, \lambda_{3}, 2 \lambda_{2}-\lambda_{3}$ or $2 \lambda_{3}-\lambda_{2}$. Hence $\mathrm{H}_{2} \mathrm{H}_{3} \mathrm{H}_{2} \mathrm{H}_{3} \backslash \mathrm{H}_{3} \mathrm{H}_{2} \mathrm{H}_{3} \mathrm{H}_{2}$ consists of $a^{2 \lambda_{2}-2 \lambda_{3}}$ only and $\mathrm{H}_{3} \mathrm{H}_{2} \mathrm{H}_{3} \mathrm{H}_{2} \backslash \mathrm{H}_{2} \mathrm{H}_{3} \mathrm{H}_{2} \mathrm{H}_{3}$ consists of $a^{2 \lambda_{3}-2 \lambda_{2}}$ only. But $H_{4} a^{2 \lambda_{2}-2 \lambda_{3}} H_{4}$ consists of $a^{\lambda}$ where

$\lambda \in\left\{2 \lambda_{2}-2 \lambda_{3}, 2 \lambda_{3}-2 \lambda_{2}\right\}$ and $a^{\lambda} t$ where $\lambda \in\left\{\lambda_{4}-2 \lambda_{2}+2 \lambda_{3}, \lambda_{4}+2 \lambda_{2}-2 \lambda_{3}\right\}$. From the symuetry between $\lambda_{2}$ and $\lambda_{3}$ above it is clear that $H_{4} a^{2 \lambda_{2}-2 \lambda_{3}} H_{4}=H_{4} a^{2 \lambda_{3}-2 \lambda_{2}} H_{4}$. Thus the sets $L$ and $R$ are equal and $G \in S P(U, V)$.

We have not tried to analyse conditions on words $u$ for which $D_{\infty} \notin S P(U, V)$ where $U=\{u\}$ and $V=\left\{u_{\sigma} \mid \sigma \in S_{n}\right\}$.

\section{REFERENCES}

[1] R.D. Blyth, 'Rewriting products of group elements [', J. Algebra 116 (1988), 506-521.

[2] M. Curzio, P. Longobardi, M. Maj and D.J.S. Robinson, 'A permutational property of groups', Arch. Math. (Basel) 44 (1985), 385-389.

[3] J.H. Evertse, 'On sums of S-units and linear recurrences', Compositio Math. 63 (1984), 225-244.

[4] I. Kaplansky, 'Groups with representations of bounded degree', Canad. J. Math. 1 (1949), 105-112.

[G] P. Longobardi, M. Maj and A.H. Rhemtılla, 'Periodic groups with permutable subgroup products', Math. Proc. Cambridge Philos. Soc. (to appear).

[0] A.H. Rheintulla and A.R. Weiss, 'Groups with permutable sulogroup products', in Group Theory: Proceedings of the 1987 Singapore Conference, Ed. K.N. Clieng and Y.K. Leong, pp. 485-495 (1)eGruyter 1989).

[7] A.H. Rhemtulla and J.S. Wilson, 'On elliptically embedded subgroups of soluble groups', Canad. J. Moth. 39 (1987), 956-968.

[8] H. Smith, 'Elliptically embedded subgroups and nilpotent products'. (submitted).

[ө] A.J. Van der Poorten, 'Additive relations in number fields', Séminaire de Thérie des Nombres de Paris, (1982-1983), 259-266..

Department of Mathematics

University of Mlberta, Edmonton

Alberta, Canada T6G 2G1 